\title{
DIAGNOSTICO AMBIENTAL FISICO-CONSERVACIONISTA DE LA SUB-CUENCA DEL RIO ANGASMARCA, LA LIBERTAD, PERÚ
}

\author{
DIAGNOSIS ENVIRONMENTAL PHYSICAL-CONSERVATIONIST IN THE SUB-BASIN \\ ANGASMARCA RIVER, LA LIBERTAD, PERÚ
}

\author{
${ }^{1}$ Jorge Luis Díaz Rimarachin
}

\begin{abstract}
Resumen
La explotación de los recursos naturales, demanda diagnósticos que aborden las necesidades de prevención de los impactos ambientales, tanto para evitar la degradación de los ambientes a ser explotados, como para minimizar la degradación ya ocurrida, proporcionando apoyo técnico para la planificación de las acciones mitigadoras. El objetivo de este trabajo fue realizar un diagnóstico ambiental físico y de conservación de suelos de la sub-cuenca del río Angasmarca en la Región La Libertad, mediante el uso de técnicas de Geoprocesamiento, sobre la base de datos topográficos e hidrográficos de cartas topográficas y de imágenes multi-espectrales CBERS y ASTER G-2 DEM de la sub-cuenca del rio Angasmarca. Los resultados de este trabajo fueron obtenidos a partir de modelos digitales de elevación (DEM), con delimitación del área de estudio en micro cuencas hidrográficas, estudio del uso y ocupación de suelos mediante la clasificación digital supervisada de imágenes de satélite CBERS, estudio de conflictos de uso, caracterización morfométrica de las micro cuencas con determinación de áreas pasibles de degradación por conflictos ambientales y de la elaboración de los mapas temáticos respectivos. Según la evaluación de uso potencial del suelo se encontró que el $85.30 \%$ de la sub-cuenca presenta aptitud para la agricultura, $13.82 \%$ presenta aptitud para pastoreo, no presenta aptitud para pastoreo/forestación y $0.89 \%$ para forestación. El $26.95 \%$ del área total de la sub-cuenca $(9,516.31$ ha), se encuentra en conflicto de uso por lo que requiere de adecuaciones que reduzcan la degradación ambiental.
\end{abstract}

Palabras clave: Cuenca hidrográfica, Geoprocesamiento, conflictos ambientales y degradación ambiental.

\begin{abstract}
The exploration of natural resources, demand diagnostics that address the prevention needs of the environmental impacts, both to avoid degradation of the environments to be exploited, to minimize degradation occurred and providing technical support for the planning of mitigation actions. The aim this study was to perform aphysical environmental diagnosis and soil conservation in the sub-basin Angasmarca river in La Libertad region, by techniques using geoprocessing, based on topographic and hydrographic data for topographic maps and multi-spectral image CBERS and ASTER DEM G-2 sub-basin. The results obtained from digital elevation models, DEM micro watershed delineation, digital classification of land use and occupation, use conflicts, morphometric characterization of micro watershed areas determining conflicts bring about environmental degradation and mapping respective thematic. The evaluation of potential land use found that $85.30 \%$ of the sub-basin is suitable for agriculture, $13.82 \%$ have aptitude for grazing, no grazing fitness/afforestation and $0.89 \%$ for afforestation. The $26.95 \%$ of the total area of the sub-basin (9516.31 ha) is in conflict requiring use of adaptations that reduce environmental degradation.
\end{abstract}

Keywords: River basin, geoprocessing, environmental conflicts and environmental degradation.

\section{Introducción}

Entre los principales factores relacionados con el aumento de la degradación del medio ambiente se tienen los cambios frecuentes no planificados en el uso del suelo por encima de la capacidad de carga del suelo (GUERRA \& CUNHA, 1996).
La erosión es una razón importante por la que los suelos agrícolas pierden su capacidad productiva. La eliminación de la vegetación nativa en una zona determinada provoca la interrupción, efímera o permanente del equilibrio natural entre el suelo y el medio ambiente (CABRAL et al., 2005). 
Según Rocha (2001), los conflictos de uso de la tierra figuran entre los principales responsables de la erosión, la sedimentación de los ríos y los embalses; afirma que es aconsejable iniciar la recuperación del ambiente a partir de la adopción de la cuenca hidrográfica como la unidad básica del planeamiento. Su adopción como unidad de gestión y planeamiento ha sido una tendencia mundial que viene ganando fuerza también en Perú.

La Ley de Recursos Hídricos No 29338 y la "Autoridad Nacional del Agua, ANA", a través del Decreto Supremo $\mathrm{N}^{\mathrm{o}}$ 039-2008-AG, establecen la cuenca hidrográfica como "unidad de gestión territorial" para definir la conformación y demarcación del área administrativa para la gestión del agua y de los recursos naturales asociados (PERÚ-ANA, 2011).

La caracterización morfométrica de una cuenca hidrográfica, es uno de los primeros y el más común de los procedimientos ejecutados en el análisis hidrológico o ambiental y tienen como objetivo aclarar las distintas cuestiones relacionadas con la comprensión de la dinámica ambiental local y regional (IOST V et al., 2007). Rocha (2001), sostiene que los parámetros que se relacionan con el deterioro o degradación del medio ambiente son la longitud de pendiente, la densidad de drenaje, la pendiente media y el coeficiente de rugosidad (RuggdenessNumber - RN). El coeficiente de rugosidad $(\mathrm{RN})$, es un parámetro que direcciona el uso potencial de las tierras rurales en micro cuencas hidrográficas en cuatro clases de coeficientes A, B, C y D, en cuanto a sus características para las actividades de agricultura, ganadería, silvicultura, reforestación o para su conservación permanente.

La evaluación permite la identificación de las potencialidades de uso y no uso, de ocupación, determinación de áreas de conflicto, sus vulnerabilidades, rendimientos futuros estimados, la optimización de las decisiones relativas a la preservación, la conservación y el desarrollo sustentable (MACEDO, 1991).

Geoprocesamiento, término amplio que a partir de la utilización de programas computacionales ofrece el uso de técnicas de procesamiento digital de imágenes de sensores remotos multi-espectrales y el uso de técnicas de sistemas de información geográfica (SIG), en la captura y manejo de información georeferenciada integrando operaciones convencionales de bases de datos con posibilidades de selección, búsqueda de información, análisis estadístico, visualización y análisis geográfico ofrecida por los mapas e imágenes (ROSA, 1996).

El objetivo del presente estudio es realizar el diagnóstico ambiental físico y de conservación de la sub-cuenca del río Angasmarca mediante la utilización de técnicas de Geoprocesamiento, siendo los objetivos específicos del estudio, el análisis y evaluación de las variables morfométricas, uso y ocupación del suelo, análisis de conflictos de uso y la degradación del medio ambiente.

\section{Materiales y método}

\section{Materiales}

Cartografía base y temática del área de estudio. Las cartas topográficas editadas por el Instituto Geográfico Nacional (IGN), en escala 1:100.000, con curvas de nivel cada 50 m, las hojas: 16-g, 16-h, 17-g y 17-h, utilizadas en el estudio de redes de drenaje, en el Modelo Digital del Terreno (MDT) y en la elaboración del mapa de pendientes.

Imágenes de sensores remotos. Uso de un mosaico de cuatro imágenes de satélite G-DEM2 del sensor ASTER: ASTGTM2: S08W078, S08W079, S09W078 y S09W079, georeferenciadas en datum WGS84, en geoide EGM96, en formato GeoTIFF, y con resolución espacial de $30 \mathrm{~m}$, fueron utilizadas en la elaboración del modelo digital del terreno (MDT), en la delimitación las micro cuencas hidrográficas, así como en la elaboración de los mapas de pendientes y redes de drenaje. Fue usada también la imagen orbital CBERS2 CCD1XS 20060721_190_109_ L2, del sensor CCD, del satélite CBERS-2, del 21 de julio de 2006, INPE del Brasil, en la elaboración del mapa de uso y ocupación del suelo.

\section{Equipamiento}

Los equipos utilizados en el desarrollo de este trabajo fueron: a) PC PENTIUM 5 con 4 GB de RAM, 500 GB de disco duro SVGA, monitor de 19", teclado, impresora de CD/DVD. b) Software: ArcGIS Desktop 9.2 (ESRI), SPRING 5.1.6 (INPE) y Microsoft Office (Excel 2007, Word 2007, etc.).

\section{Método}

El estudio del diagnóstico ambiental físicoconservacionista comprende la identificación, modelamiento matemático y cuantificación de los daños ambientales ocasionados por el uso inadecuado de los suelos de la sub-cuenca del río Angasmarca, a través de la evaluación de las variables morfométricas de las micro cuencas, el análisis de conflictos de uso del suelo y la degradación del medio ambiente. El desarrollo metodológico fue realizado mediante la aplicación de las técnicas de geoprocesamiento, entre ellas el procesamiento digital y gestión de datos de las imágenes orbitales antes indicadas, el uso de los MDTs a partir de cartas topográficas e imágenes y uso de mapas temáticos, mediante la aplicación del software SPRIG 1.3.6 y de la interface de Sistema de Información Geográfica SIGArcGIS 9.2, ejecutado en el orden siguiente.

Modelo digital del terreno (MDT).- Fue elaborado a partir de un mosaico de cartas topográficas, también se elaboró un MDT con mayor precisión a partir de imágenes satelitales ASTER G-DEM2, luego de la transformación de coordenadas geográficas (Datum WGS-84) a coordenadas UTM (Datum SIRGAS), se hizo el recorte 
vectorial ajustado al área de estudio. Los MDTs fueron elaborados mediante las herramientas de 3D-Analist de ArcGIS (TINs) y el recorte vectorial mediante "extracción por máscaras". Los niveles altimétricos de TINs fueron clasificados en 11 clases con intervalos de $250 \mathrm{~m}$.

Delimitación de micro cuencas hidrográficas.La delimitación de micro cuencas de la sub-cuenca hidrográfica del rio Angasmarca se realizó mediante uso de las herramientas "Watershed Delineation Tools" del programa FWD a partir del MDE, con datos de entrada y umbral proporcionado por el usuario (el valor de la referencia se basó en la resolución del MDT y la topografía), el resultado fue la delimitación de las micro cuencas hidrográficas.

Obtención de las variables morfométricas de las micro cuencas.- Las variables morfométricas correlacionadas con la geometría, la red de drenaje y la topografía de la sub-cuenca fueron determinadas para cada una de las micro cuencas del área de estudio entre ellas: el área (A) y perímetro $(P)$, la longitud de red de drenaje $(\mathrm{Cr})$, la densidad de drenaje (Dd), la pendiente media $(\mathrm{H})$ y el coeficiente de rugosidad (RN), fueron determinadas de la siguiente manera:

Área (A).- El área como elemento básico para el cálculo de los diversos índices morfométricos (TONELLO, 2005), por lo general se expresa en $\mathrm{km}^{2}$. El cálculo del área de las micro cuencas fue realizado desde la tabla de atributos de ArcGIS, mediante el uso de las herramientas "field calculator".

Perímetro (P).- Determinado por la medida de la longitud de la línea perimétrica de las micro cuencas hidrográficas, desde la tabla de atributos de ArcGIS, expresado en km,

Longitud total de la red de drenaje $(\mathbf{C t})$.- Corresponde al cálculo de la longitud total de cursos de agua de la red de drenaje de cada micro cuenca, mediante las herramientas de "field calculator" desde la tabla de atributos, expresada en $\mathrm{km}$.

Longitud total de cursos por orden (Ctw).- Longitud total de los cursos en cada orden, siendo identificados por $\mathrm{Ctw}$ (Ctw1, Ctw2, etc.). El cálculo fue realizado mediante el software MS Excel, a partir de la tabla de atributos de la red de drenaje de cada una de las micro cuencas del área de estudio.

Densidad de drenaje (Dd).- Es un indicador importante del grado de desarrollo del sistema de drenaje de una micro cuenca e indica la eficacia del drenaje

(HORTON, 1945). El cálculo realizado fue mediante la ecuación 2.1
$\mathrm{Dd}=\mathrm{Ct} / \mathrm{A}$

donde $\mathrm{Dd}=$ densidad de drenaje $\left(\mathrm{km} \cdot \mathrm{km}^{-2}\right), \mathrm{Ct}=$ longitud total de todos los cursos de drenaje $(\mathrm{km})$, se incluyen tanto los cursos perennes como los temporales y A = área de drenaje $\left(\mathrm{km}^{2}\right)$. Según Vilela \& Mattos (1975), el índice de la Dd varía de $0.5 \mathrm{~km} . \mathrm{km}^{-2}$, para cuencas con drenaje pobre a $3.5 \mathrm{~km} . \mathrm{km}^{-2} \mathrm{o}$ más para cuencas excepcionalmente bien drenadas.

Pendiente media (H).- Expresada en porcentaje (\%), el cálculo fue mediante la aplicación de la ecuación 2.2, desde la tabla de atributos del "shapefile" de curvas de nivel generado en ArcGIS y finalmente mediante la ayuda del software MS Excel.

$\mathrm{H}=\left(\left(\sum \mathrm{LCN} . \Delta \mathrm{h}\right) / \mathrm{A}\right) * 100$

donde, $\mathrm{H}=$ pendiente media $(\%)$,= sumatoria de las longitudes de todas las curvas

de nivel de las micro cuencas mapeadas (m) por el área $\mathrm{A}$ $=$ área de la micro cuenca $\left(\mathrm{m}^{2}\right)$ y la $=$ equidistancia de las curvas de nivel (m).

Coeficiente de rugosidad (Número Ruggdeness RN).- Es el parámetro adimensional que direcciona el uso potencial de tierras rurales en relación con las características para la agricultura, pastoreo y/o forestación o para su preservación permanente, según la Tabla 2. El cálculo se realizó mediante la aplicando de la ecuación 2.3 y uso del software MS Excel.

$\mathrm{RN}=\mathrm{Dd} \cdot \mathrm{DME}$

donde $\mathrm{RN}=$ coeficiente de rugosidad, $\mathrm{Dd}=$ densidad de drenaje $\left(\mathrm{km}^{\mathrm{k}} \mathrm{km}^{-2}\right)$ y $\mathrm{H}=$ pendiente media de la micro cuenca en $(\%)$.

Clasificación de uso potencial del suelo (UP). El uso potencial del suelo en micro cuencas hidrográficas es determinado en cuatro clases según Sicco Smith y citado por Rocha \& Hurtz (2001), según el procedimiento siguiente:

1) Determinación de la amplitud de variación (A) y rango de clase (I), mediante las ecuaciones 2.4 y 2.5 donde el denominador (4) representa el número de clases de aptitud (A, B, C, D).

$\mathrm{A}=(>$ valorRN $)-(<$ valorRN $)$

$$
\mathrm{I}=(\mathrm{A} / 4)
$$


2) Los intervalos de dominio se inician en la clase inferior (menor valor de RN), se incrementa el valor del intervalo de clase adicionando un rango de clase.

3) Elaboración del mapa de uso potencial del suelo, según la Tabla 1.

4) Si el uso potencial de suelos es de clase A, se debe asignar el valor ponderado 10 (peor situación ambiental), representa el mayor valor a ser pagado en pasivo ambiental y el valor de 1 (mejor situación ambiental).

Tabla 1. Clasificación de Uso potencial de Suelos y valores ponderados.

\begin{tabular}{rlc}
\hline $\begin{array}{c}\text { Clase } \\
\text { RN }\end{array}$ & Uso potencial de la tierra (UP) & $\begin{array}{c}\text { Valores } \\
\text { ponderados }\end{array}$ \\
\hline A & $\begin{array}{l}\text { Suelos con aptitud para la } \\
\text { agricultura (RN menor) }\end{array}$ & 10 \\
B & $\begin{array}{l}\text { Suelos con aptitud para el } \\
\text { pastoreo }\end{array}$ & 7 \\
C & $\begin{array}{l}\text { Suelos con aptitud para el } \\
\text { pastoreo / forestación }\end{array}$ \\
D $\quad \begin{array}{l}\text { Suelos con aptitud para la } \\
\text { forestación (RN más alto) }\end{array}$ & 4 \\
\hline
\end{tabular}

Elaboración de los mapas de pendientes y red de drenaje.- Fueron obtenidos a partir de las curvas de nivel (CN) y de la red hidrográfica de las micro cuencas, en formato Shapefile, mediante las herramientas "extracción por máscaras" y el uso de las tablas de atributos del sistema ArcGIS.

Mapa de uso y ocupación del suelo (MUO). Obtenido por clasificación digital supervisada de la imagen CBERS-2, mediante el uso del software SPRING 5.1.6, según las clases de uso mostradas en la Tabla 3.

\section{Evaluación ambiental físico-conservacionista de las micro cuencas}

1) Determinación de conflictos de uso del suelo.Realizada mediante el uso de la técnica de superposición de los mapas de uso potencial del suelo (UP) y él de uso y ocupación del suelo (MUO), así como del uso de la Tabla 2 (clasificación por diferencia de pesos). Las alternativas de clases de conflictos de uso del suelo que presentan significancia son seis, de los cuales sólo cuatro fueron encontrados en este estudio:

2-1: Conflicto clase $1 \Rightarrow$ Pastizal x Agricultura.

3-1: Conflicto clase $2 \Rightarrow$ Pastizal / Forestación $\mathrm{x}$ Agricultura.

4-1: Conflicto clase $3 \Rightarrow$ Forestación x Agricultura.

4-2: Conflicto clase $2 \Rightarrow$ Forestación $x$ Pastizal.
Tabla 2. Clasificación de pesos referentes a las clases de RN y Uso Actual.

\begin{tabular}{lll}
\hline Clases de UP & Uso Actual & Peso \\
\hline Agricultura & Clase A & 1 \\
Pastizal & Clase B & 2 \\
Pastizal/Forestal & Clase C & 3 \\
Forestal & Clase D & 4 \\
\hline Fuente: Adaptado de Farías do Valle (2008)
\end{tabular}

2) Áreas a reforestar.- Según Rocha (2001), en micro cuencas con pendientes medias de hasta $15 \%$, el reforestamiento mínimo necesario es del $25 \%$, sin embargo, en pendientes medias mayores al $15 \%$ el reforestamiento mínimo será del $50 \%$, es decir, el autor considera que la cobertura forestal en este porcentaje representa la cubertura forestal mínima que garantiza la producción de la biomasa superficial necesaria para la preservación de los recursos hídricos, la flora, la fauna y la conservación de suelos, para satisfacer las necesidades en términos económicos, energéticos y ecológicos.

3) Exceso o disponibilidad de áreas en agricultura en las micro cuencas.- En micro cuencas de clase A, la disponibilidad de tierras para la agricultura se determina por la diferencia entre el área total de la micro cuenca y la suma de las áreas de floresta, agricultura, agua y áreas a reforestar. En micro cuencas de clases B, C y $\mathrm{D}$, las áreas disponibles son sólo aquellas destinadas para la agricultura de estas micro cuencas.

4) Áreas a ser trabajadas para el manejo correcto de la micro cuenca.- Para las micro cuencas de clase A las áreas a ser trabajadas son las áreas de conflictos, áreas a reforestar y áreas disponibles para agricultura. Para las micro cuencas de clases B, C y D, las áreas a ser trabajadas son las áreas destinadas a reforestación y las disponibles para agricultura.

5) Áreas degradadas por micro cuenca.- El grado de deterioro o degradación de las micro cuencas se determinó mediante la suma de las áreas de conflicto y las áreas a ser reforestadas.

6) Porcentaje de degradación de cada micro cuenca.El porcentaje (\%) de degradación de una micro cuenca fue determinada mediante la relación entre el área de deterioro y el área total de la micro cuenca multiplicada por 100.

7) Las prioridades para el manejo de micro cuencas.Las prioridades de manejo de las micro cuencas se determinaron sobre la base del porcentaje de degradación de las micro cuencas. La micro cuenca que tiene el mayor valor corresponde a la prioridad 1 y en la medida que el valor de degradación disminuye, disminuye también la prioridad de manejo. 
8) Códigos y criterio de estratificación.- El diagnóstico físico-conservacionista se realizó a través de 7 indicadores ambientales, se trabajó con el valor medio encontrado y los valores mínimo y máximo de cada indicador. El valor mínimo es 1 y el valor máximo es 10, el valor mínimo (1) corresponde a la mejor situación ambiental y el valor máximo (10), corresponde a la peor situación ambiental. Estos datos son necesarios para determinar del modelo matemático que define la degradación ambiental de la sub-cuenca.

9) Determinación del modelo matemático de la degradación ambiental.- El modelo matemático para evaluar la degradación ambiental físicoconservacionista de las micro cuencas en estudio es la ecuación de la recta que define la degradación ambiental de la sub-cuenca, donde el valor de y varía de 0 (cero) a $100 \%$ de degradación.

$$
\mathrm{y}=\mathrm{ax}+\mathrm{b}
$$

\section{Resultados y discusión}

El modelo digital del terreno (MDT).- Fue obtenido a partir de las imágenes ASTER G-DEM2, como se muestra en la Figura 1. En el MDT se puede apreciar la variación de la altitud donde las menores cotas varían en torno a $1,980 \mathrm{~m}$ y las mayores en torno a 4,600 m

Delimitación de micro cuencas hidrográficas.- La sub-cuenca del rio Angasmarca fue dividida en 11 micro cuencas hidrográficas como se muestra en el mapa de uso potencial del suelo por micro cuencas, Figura 2, como resultado de la aplicación de la técnica de delimitación de micro cuencas mediante el uso del programa "Watershed Delineation Tools" (FWD).

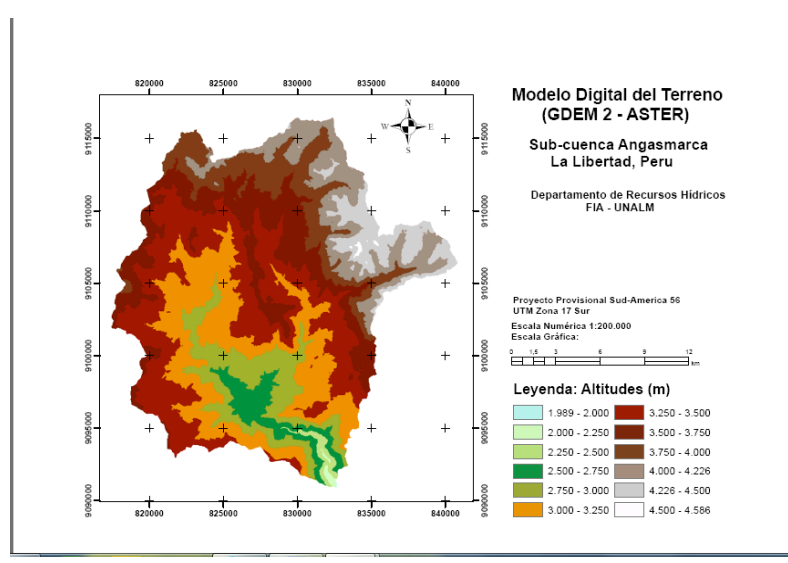

Figura 1. Altimetría - Modelo Digital del Terreno. SIGArcGIS 9.2.

Análisis morfométrico de la sub-cuenca del río Angasmarca.- Según el análisis del sistema de drenaje de la sub-cuenca del río Angasmarca es una cuenca de quinto orden, lo cual indica tener una ramificación media, con un área de $353.075 \mathrm{~km}^{2}$ y perímetro de $96.04 \mathrm{~km}$. La longitud del curso principal es de $32.29 \mathrm{~km}$, con una red de drenaje total de $176.264 \mathrm{~km}$. Observando los valores medios de las pendientes, verificamos que la inclinación de los interfluvios en las micro cuencas presenta diferencias significativas de $28.05 \%$ a $50.69 \%$, siendo la pendiente media de $33.89 \%$ para la sub-cuenca. En general los valores de las variables morfométricas de las micro cuencas indican estar sujetas a fenómenos erosivos relacionados a condiciones de pendientes medias altas, de suelo expuesto y de áreas con escasa vegetación permanente, como se muestran en las Tablas 3, 4, 5 y los mapas de uso y ocupación del suelo (actual y potencial), en las Figuras 3 y 4, respectivamente.

Tabla 3 . Variables morfométricas correlacionadas con la geometría, la red de drenaje y el relieve de las micro cuencas del río Angasmarca.

\begin{tabular}{|c|c|c|c|c|c|c|c|}
\hline $\begin{array}{l}\text { Micro } \\
\text { cuenca }\end{array}$ & $\begin{array}{c}\text { Área } \\
\mathrm{A} \\
\left(\mathrm{km}^{2}\right)\end{array}$ & $\begin{array}{c}\text { Perímetro } \\
\mathrm{P} \\
(\mathrm{km})\end{array}$ & $\begin{array}{l}\text { Longitud total } \\
\text { de drenaje } \\
\text { Cr } \\
(\mathrm{km})\end{array}$ & $\begin{array}{l}\text { Densidad de } \\
\text { Drenaje } \\
\text { Dd } \\
\left(\mathrm{km} \cdot \mathrm{km}^{-2}\right)\end{array}$ & $\begin{array}{c}\text { Pendiente } \\
\text { S } \\
(\%)\end{array}$ & $\begin{array}{c}\text { Coeficiente } \\
\text { Rugosidad } \\
\text { Rn } \\
\text { (Adim) }\end{array}$ & $\begin{array}{l}\text { Altitud } \\
\qquad \begin{array}{c}\text { h } \\
(\mathrm{m})\end{array}\end{array}$ \\
\hline 1 & 52.867 & 34.240 & 30.074 & 0.569 & 31.942 & 18.175 & 3700 \\
\hline 2 & 25.922 & 21.375 & 19.454 & 0.750 & 29.234 & 21.926 & 3900 \\
\hline 3 & 19.793 & 23.840 & 7.596 & 0.384 & 28.048 & 10.770 & 3600 \\
\hline 4 & 31.205 & 30.241 & 18.420 & 0.590 & 28.548 & 16.843 & 4150 \\
\hline 5 & 22.257 & 23.969 & 12.085 & 0.543 & 36.382 & 19.755 & 4150 \\
\hline 6 & 66.140 & 38.295 & 34.653 & 0.524 & 33.539 & 17.574 & 4500 \\
\hline 7 & 15.436 & 19.062 & 12.223 & 0.792 & 49.436 & 39.153 & 4650 \\
\hline 8 & 33.357 & 27.568 & 19.472 & 0.584 & 50.282 & 29.365 & 4500 \\
\hline 9 & 3.126 & 8.072 & 5.062 & 1.619 & 50.687 & 82.062 & 3960 \\
\hline 10 & 46.016 & 30.434 & 23.445 & 0.509 & 35.340 & 17.988 & 4340 \\
\hline 11 & 36.957 & 33.228 & 15.599 & 0.422 & 50.229 & 21.197 & 3750 \\
\hline Media & 32.098 & 26.393 & 18.008 & 0.662 & 38.515 & 26.801 & 4109.091 \\
\hline
\end{tabular}


La densidad media de drenaje de la sub-cuenca es de 0.662 $\mathrm{km} / \mathrm{km}^{2}$, indica que ésta posee baja capacidad de drenaje, sin embargo, en las micro cuencas ocurren variaciones de 0.422 a $1.619 \mathrm{~km} / \mathrm{km}^{2}$, según Villela y Mattos (1975), se trata de micro cuencas bien drenadas.

Clasificación de uso potencial del suelo.- Los resultados de la clasificación de uso potencial de suelos de la subcuenca Angasmarca mostrados en la Figura 2 y en la

Tabla 4, indican que el $85.30 \%$ del área es de uso potencial para agricultura, mientras que $13.82 \%$ es para pastoreo, no presenta aptitud para pastoreo/forestación y sólo el $0.89 \%$ es para forestación.

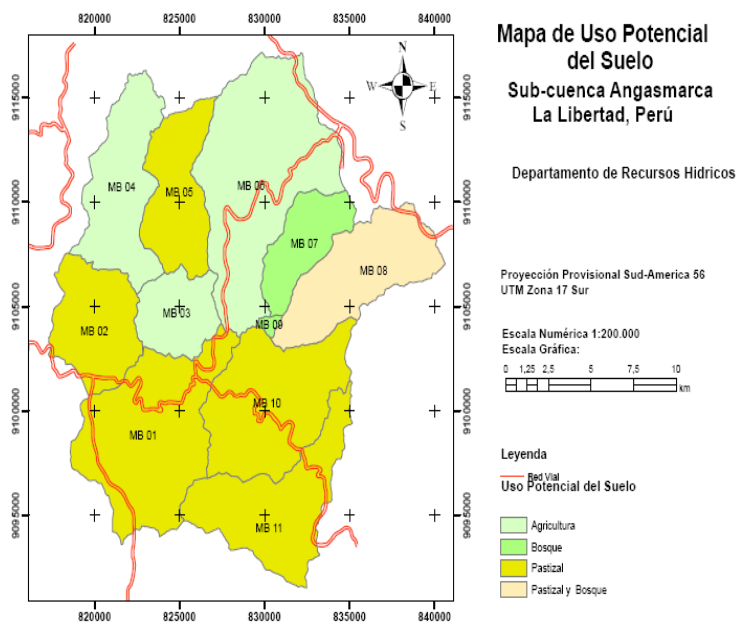

Figura 2. Mapa de Clasificación del uso potencial del suelo.

Se verifica que el mayor uso potencial del suelo se refiere a la agricultura y al pastoreo, haciendo un total de 99.12 $\%$, resultado obtenido por correlación del uso potencial del suelo con el grado de pendiente y las características de la red de drenaje.
Tabla 4. Clasificación de uso potencial del suelo y variables morfométricas.

\begin{tabular}{cccccc}
\hline $\begin{array}{c}\text { Clases } \\
\text { de RN cuenca }\end{array}$ & $\begin{array}{c}\text { Área } \\
\text { ha }\end{array}$ & $\begin{array}{c}\mathrm{H} \\
(\%)\end{array}$ & $\begin{array}{c}\mathrm{D} \\
(\mathrm{km} / \mathrm{ha})\end{array}$ & $\begin{array}{c}R N \times 10^{\mathrm{n}=1 \mathrm{a} 8} \\
\mathrm{adim} .\end{array}$ \\
\hline A & 1 & $5,286.74$ & 31.942 & 0.00569 & 18.175 \\
$\mathrm{~A}$ & 2 & $2,592.18$ & 29.234 & 0.00750 & 21.926 \\
$\mathrm{~A}$ & 3 & $1,979.26$ & 28.048 & 0.00384 & 10.770 \\
$\mathrm{~A}$ & 4 & $3,120.53$ & 28.548 & 0.00590 & 16.843 \\
$\mathrm{~A}$ & 5 & $2,225.71$ & 36.382 & 0.00543 & 19.755 \\
$\mathrm{~A}$ & 6 & $6,613.96$ & 33.539 & 0.00524 & 17.574 \\
$\mathrm{~B}$ & 7 & $1,543.58$ & 49.436 & 0.00792 & 39.153 \\
$\mathrm{~B}$ & 8 & $3,335.67$ & 50.282 & 0.00584 & 29.365 \\
$\mathrm{D}$ & 9 & 312.63 & 50.687 & 0.01619 & 82.062 \\
$\mathrm{~A}$ & 10 & $4,601.60$ & 35.340 & 0.00509 & 17.988 \\
$\mathrm{~A}$ & 11 & $3,695.67$ & 50.229 & 0.00422 & 21.197 \\
\hline Total & --- & $35,307.5338 .51518$ & 0.006624 & 26.801 \\
\hline
\end{tabular}

Clasificación de uso y ocupación del suelo.- Los resultados de la clasificación de uso y ocupación del suelo de la sub-cuenca Angasmarca, se muestra en la Tabla 5 y en la Figura 3, e indican que la agricultura ocupa 30.6\% del área de la sub-cuenca (10,798.87 ha), de las cuales el $3.3 \%$ corresponde a áreas clasificadas como "agricultura irrigada", el $13.45 \%$ es "agricultura de secano" y 10.9 $\%$ corresponde a una asociación de agricultura-pastoreo (cultivos anuales intercalados con pastos mejorados).
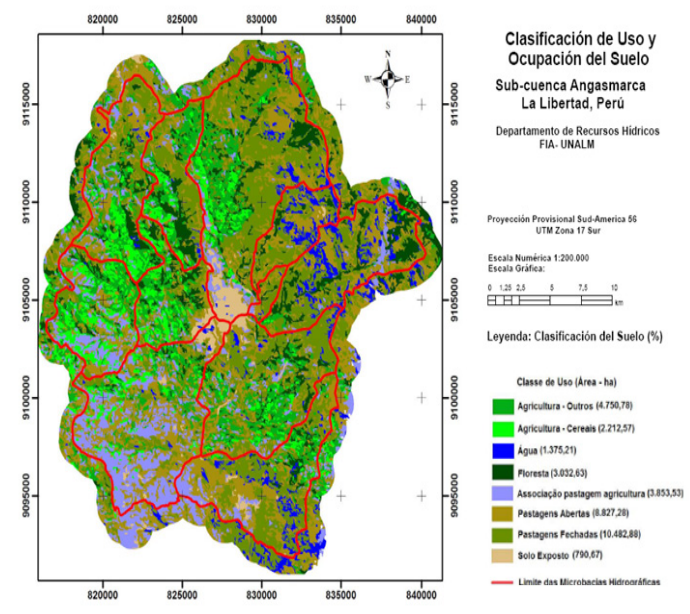

Figura 3. Mapa de uso y ocupación del suelo. SPRING 5.1.6 (INPE).

Tabla 5. Clasificación de uso y ocupación del suelo de la sub-cuenca Angasmarca.

\begin{tabular}{|c|c|c|c|c|c|c|c|c|c|}
\hline $\begin{array}{l}\text { Micro } \\
\text { cuenca }\end{array}$ & Agua & $\begin{array}{c}\text { Suelo } \\
\text { Expuesto }\end{array}$ & Forestal & $\begin{array}{l}\text { Agricultura } \\
\text { otros }\end{array}$ & $\begin{array}{l}\text { Pastizal } \\
\text { Abierto }\end{array}$ & $\begin{array}{l}\text { Pastizal } \\
\text { cerrado }\end{array}$ & $\begin{array}{c}\text { Agricultura } \\
\text { cereales }\end{array}$ & $\begin{array}{l}\text { Asociación } \\
\text { Agric-past. }\end{array}$ & Área tota \\
\hline & (ha) & (ha) & (ha) & (ha) & (ha) & (ha) & (ha) & (ha) & (ha) \\
\hline 1 & 41.08 & 205.14 & 93.17 & 586.15 & $1,707.20$ & 631.74 & 324.54 & $1,697.72$ & $5,286.74$ \\
\hline 2 & 1.79 & 0.00 & 138.85 & 584.81 & 540.14 & 253.90 & 567.22 & 505.46 & $2,592.18$ \\
\hline 3 & 17.53 & 7.00 & 193.60 & 377.18 & 494.09 & 503.95 & 207.29 & 178.61 & $1,979.26$ \\
\hline 4 & 27.64 & 11.03 & 305.24 & 594.67 & 779.00 & 794.54 & 326.81 & 281.61 & $3,120.53$ \\
\hline 5 & 19.34 & 0.00 & 365.67 & 514.95 & 357.21 & 673.16 & 186.29 & 109.07 & $2,225.71$ \\
\hline 6 & 188.04 & 359.20 & 706.51 & 843.76 & $1,237.74$ & $2,666.93$ & 329.76 & 282.02 & $6,613.96$ \\
\hline 7 & 276.63 & 11.36 & 114.39 & 12.19 & 490.51 & 629.44 & 0.00 & 9.06 & $1,543.58$ \\
\hline 8 & 460.52 & 0.00 & 557.68 & 66.33 & 865.77 & $1,259.72$ & 8.61 & 117.04 & $3,335.67$ \\
\hline 9 & 4.76 & 4.16 & 22.06 & 3.68 & 93.28 & 170.35 & 0.00 & 14.33 & 312.63 \\
\hline 10 & 104.71 & 108.07 & 368.71 & 899.87 & $1,056.79$ & $1,564.62$ & 192.36 & 306.47 & $4,601.60$ \\
\hline 11 & 233.16 & 84.69 & 166.75 & 267.17 & $1,205.55$ & $1,334.54$ & 69.68 & 334.13 & $3,695.67$ \\
\hline Total & $1,375.21$ & 790.67 & $3,032.63$ & $4,750.78$ & $8,827.28$ & $10,482.88$ & $2,212.57$ & $3,835.53$ & $35,307.53$ \\
\hline
\end{tabular}


Los pastizales ocupan $54.7 \%$ del área de la sub-cuenca (19,310.16 ha), siendo $29.7 \%$ áreas de pastos naturales densos (pastizal cerrado) y $25 \%$ corresponde a pastos naturales intercalados con formaciones rocosas y de suelo expuesto (pastizal abierto).

Los bosques naturales y plantados ocupan $8.6 \%$ de la sub-cuenca $(3,032.63 \mathrm{ha})$, suelo expuesto (sin cobertura), ocupa $2.24 \%$ de la sub-cuenca (790.67 ha), esta categoría comprende a tierras en descanso, barbecho y tierras destinadas a la minería, etc.) y finalmente, el 3.9 $\%$ corresponde a cuerpos de agua $(1,375.21 \mathrm{ha})$, entre lagunas y humedales.

Áreas de conflicto de uso en la sub-cuenca.- De acuerdo con los resultados mostrados de la Tabla 5, de 35,307.53 ha, area total de la sub-cuenca, se deduce que:

1) El $26.95 \%$ del área $(9,516.31 \mathrm{ha})$, se encuentra como área en conflicto, siendo $85.30 \%(30,115.65$ ha), áreas de conflicto de clase 1 , es decir, que existen áreas de pastoreo que están siendo utilizadas en agricultura, con pendientes superiores al $10 \%$ y que su uso en esta clase requiere aplicación de medidas de conservación de suelos.

2) El $13.82 \%$ del área (4,879.25 ha), corresponde a áreas de conflicto de clase 2 , es decir, que áreas destinadas a pastoreo, están siendo utilizadas para agricultura, se incluyen las áreas desérticas (áreas quemadas) y asociaciones de uso pastizal-agricultura.

3) No hay micro cuencas de clase C, es decir, no hay áreas de conflicto de clase 2, por tanto no hay áreas de pastoreo/forestación, que estén siendo utilizadas en agricultura.

4) El $0.88 \%$ de la superficie (312.63 ha), corresponde a áreas de conflicto de clase 3 , lo cual significa que áreas de forestación están siendo utilizados en pastoreo, agricultura, áreas desérticas (áreas quemadas) y asociaciones de pastizal-agricultura.

Áreas a forestar.- De acuerdo con la metodología desarrollada por Rocha (2001), las micro cuencas del río Angasmarca tienen pendientes promedio mayores de $15 \%$, por tanto el forestamiento mínimo necesario de las micro cuencas para protegerlas contra la erosión y contra los efectos de la sequía será de $50 \%$. El área total a ser forestada es de $14,622.08$ ha, de la cual 86.99 $\%(12,720.29$ ha), está localizada en micro cuencas de uso potencial clase A; $12.09 \%$ (1,767.52 ha), en micro cuencas de clase B y $0.92 \%$ (134.27 ha), en micro cuencas de clase $\mathrm{D}$, no existiendo micro cuencas de clase C, como se muestra en la Tabla 6 .
Disponibilidad y/o exceso en agricultura.- La disponibilidad de tierras para la agricultura es de 9,083.00 ha, de las cuales $99.00 \%$ (8,992.19 ha), se encuentran en micro cuencas de clase A, $0.96 \%$ (87.13 ha), en micro cuencas de clase B y $0.04 \%$ (3.68 ha), en micro cuencas de clase D.

Áreas a ser trabajadas para el manejo correcto de cada micro cuenca.- Del área total de tierras a ser trabajadas para la gestión correcta de las micro cuencas es de 29,913.59 ha, como se muestran en la Tabla 6. El 93.34 $\%(27,920$ ha $)$, de tierras se encuentran en micro cuencas de clase A, $6.20 \%$ (1854.65 ha), son de clase B y el 0.46 $\%$ (137.95 ha), son micro cuencas de clase D.

Áreas degradadas por micro cuenca.- De la evaluación de los resultados, según la Tabla 6, y la Figura 4, se sabe que el área total de degradación ambiental es de 24,138.39 ha, de la cual el $84.39 \%$ (20,369.93 ha), pertenece a micro cuencas de clase A, el $13.87 \%$ (3,348.39 ha), se encuentra en micro cuencas de clase B y el $1.74 \%$ (420.07 ha), en clase D.

Porcentaje de degradación de cada micro cuenca.- El porcentaje medio de degradación de las micro cuencas se encuentra distribuido en: $68.91 \%$ para las 8 micro cuencas de clase A (micro cuencas enumeradas de 1 al 6 , 10 y 11 ), con el $70.74 \%$ de degradación para las 2 micro cuencas de clase B (micro cuencas 7 y 8 ) y de $100 \%$ para la micro cuenca 9 de clase D. Se atribuye el $100 \%$ de degradación para esta última micro cuenca (porcentaje máximo de degradación), en medida que el porcentaje calculado resultó mayor de $100 \%$.

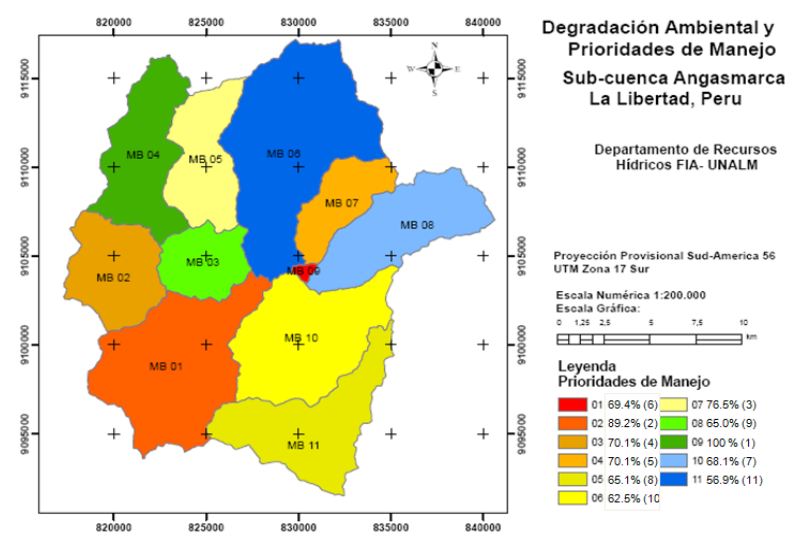

Figura 4. Mapa de degradación ambiental y prioridades de manejo. 
Tabla 6. Conflictos por micro cuenca evaluación físico-conservacionista de la sub-cuenca del rio Angasmarca.

\begin{tabular}{|c|c|c|c|c|c|c|c|c|c|c|c|c|c|c|}
\hline \multirow[t]{2}{*}{$\begin{array}{l}\text { Clases } \\
\text { de RN }\end{array}$} & \multirow[t]{2}{*}{$\begin{array}{l}\text { Micro } \\
\text { Cuenca }\end{array}$} & \multirow{2}{*}{$\begin{array}{c}\text { Área M.C } \\
\text { (ha) }\end{array}$} & \multicolumn{2}{|c|}{ Conflictos de Uso } & \multirow{2}{*}{$\begin{array}{c}\text { Bosques } \\
(\%) \\
\end{array}$} & \multicolumn{2}{|c|}{ Área a Forestar } & \multicolumn{2}{|c|}{$\begin{array}{l}\text { Disp y/o exceso } \\
\text { enagricultura }\end{array}$} & \multicolumn{2}{|c|}{$\begin{array}{l}\text { Área a ser trabajada para } \\
\text { manejo correcto de micro } \\
\text { cuenca }\end{array}$} & \multirow{2}{*}{$\begin{array}{l}\text { Área degr. por } \\
\text { micro cuenca }\end{array}$} & \multirow{2}{*}{$\begin{array}{c}\% \text { de degr. por } \\
\text { micro cuenca } \\
(\%)\end{array}$} & \multirow{2}{*}{$\begin{array}{l}\text { Prioridades } \\
\text { (adim) }\end{array}$} \\
\hline & & & (ha) & $\%$ & & (ha) & $(\%)$ & (ha) & $(\%)$ & (ha) & $(\%)$ & & & \\
\hline $\mathrm{O} 1$ & $\mathrm{O} 2$ & $\mathrm{O} 3$ & 4 & 5 & 6 & 7 & 8 & 9 & 10 & 11 & 12 & 13 & 14 & 15 \\
\hline A & 1 & $5,286.74$ & $1,115.83$ & 21.11 & 1.76 & $2,550.20$ & 48.24 & 1690.95 & 31.98 & 5357.63 & 100 & $3,666.68$ & 69.36 & 6 \\
\hline A & 2 & $2,592.18$ & $1,154.03$ & 44.52 & 5.36 & $1,157.24$ & 44.64 & 142.36 & 5.49 & 2453.54 & 94.65 & $2,311.18$ & 89.16 & 2 \\
\hline A & 3 & $1,979.26$ & 591.47 & 29.88 & 9.78 & 796.03 & 40.22 & 387.6 & 19.58 & 1775.13 & 89.69 & $1,387.53$ & 70.1 & 4 \\
\hline $\mathrm{A}$ & 4 & $3,120.53$ & 932.51 & 29.88 & 9.78 & $1,255.03$ & 40.22 & 611.09 & 19.58 & 2798.68 & 89.69 & $2,187.59$ & 70.1 & 5 \\
\hline $\mathrm{A}$ & 5 & $2,225.71$ & 701.24 & 31.51 & 16.43 & 747.17 & 33.57 & 392.29 & 17.62 & 1840.7 & 82.7 & $1,448.41$ & 65.08 & 8 \\
\hline A & 6 & $6,613.96$ & $1,532.72$ & 23.17 & 10.68 & $2,600.61$ & 39.32 & $1,945.28$ & 29.41 & $6,078.61$ & 91.91 & $4,133.33$ & 62.49 & 10 \\
\hline B & 7 & $1,543.58$ & 523.12 & 33.89 & 7.41 & 657.41 & 42.59 & 12.19 & 0.79 & 669.6 & 43.38 & $1,180.53$ & 76.48 & 3 \\
\hline B & 8 & $3,335.67$ & $1,057.75$ & 31.71 & 16.72 & $1,110.11$ & 33.28 & 74.94 & 2.25 & $1,185.05$ & 35.53 & $2,167.86$ & 64.99 & 9 \\
\hline $\mathrm{D}$ & 9 & 312.63 & 285.8 & 91.42 & 7.06 & 134.27 & 42.95 & 3.68 & 1.18 & 137.95 & 44.13 & 420.07 & 100 & 1 \\
\hline A & 10 & $4,601.60$ & $1,200.30$ & 26.08 & 8.01 & $1,932.21$ & 41.99 & $1,103.74$ & 23.99 & 4236.25 & 92.06 & $3,132.51$ & 68.07 & 7 \\
\hline A & 11 & $3,695.67$ & 421.54 & 11.41 & 4.51 & $1,681.16$ & 45.49 & 1277.75 & 34.57 & 3380.45 & 91.47 & $2,102.70$ & 56.9 & 11 \\
\hline Total & & $35,307.53$ & $9,516.31$ & 375 & 97.5 & $14,622.08$ & 453 & $9,083.00$ & 186.4 & $29,913.59$ & 856.55 & $24,138.39$ & 827.1 & 66 \\
\hline Média & & $3,209.78$ & 865.12 & 34.1 & 8.86 & $1,329.28$ & 41.1 & 825.73 & 16.95 & 2719.42 & 77.87 & $2,194.40$ & 75.19 & 6 \\
\hline
\end{tabular}

LEYENDA DEL USO DE LA TIERRA: Conflicto en A: Agricultura + áreas quemadas; Conflicto en B: Agricultura + asociaciones+ áreas quemadas

Conflicto en C: Agricultura + asociaciones+ áreas quemadas; Conflicto en D: Pastoreo + Agricultura + asociaciones + deforestación + áreas quemadas 
Prioridades para la gestión de las micro cuencas hidrográficas.- De acuerdo con los resultados del porcentaje de degradación por micro cuencas mostrados en la Tabla 6 y en el mapa de degradación ambiental y prioridades de manejo, según la Figura 4, la prioridad de manejo número (1), la tiene la micro cuenca 9 de clase $\mathrm{D}$, por tener el mayor porcentaje de degradación de aproximadamente $100 \%$, finalmente, la última prioridad de manejo (11), corresponde a la micro cuenca 11 de clase A, por tener el menor porcentaje de degradación ambiental.

\section{Diagnóstico físico-conservacionista, códigos y criterios}

de estratificación.- De los resultados obtenidos, mediante la aplicación de códigos y uso de criterios de estratificación, los valores significativos encontrados según los 7 indicadores ambientales analizados encontramos que el $35 \%$ del área total de la sub-cuenca corresponde a áreas en conflicto, el 17\% a áreas disponibles para agricultura, sólo el $9 \%$ corresponde a áreas con cobertura forestal, se indica además que el área a forestar debe ser en el orden del $42 \%$ como medida de conservación ambiental, entre otros indicadores ambientales que son mostrados en la Tabla 7. El factor físico-conservacionista total encontrado fue de 38 , sobre un valor total máximo posible de 70 y un valor total mínimo de 7 .

Tabla 7. Valores significativos: encontrados, mínimos y máximos.

\begin{tabular}{|c|c|c|c|c|c|}
\hline \multirow[b]{2}{*}{ Códigos } & \multirow[b]{2}{*}{ Indicadores ambientales } & \multirow[b]{2}{*}{$\%$} & \multicolumn{3}{|c|}{ Valores significativos } \\
\hline & & & Encontrado & Mínimo & Máximo \\
\hline 1 & Conflictos de uso & 35 & 4 & 1 & 10 \\
\hline 2 & Cobertura forestal & 9 & 9 & 1 & 10 \\
\hline 3 & Área a forestar & 42 & 5 & 1 & 10 \\
\hline 4 & Disponibilidad en áreas para agricultura & 17 & 2 & 1 & 10 \\
\hline 5 & Exceso en áreas para agricultura & 17 & 2 & 1 & 10 \\
\hline 6 & $\begin{array}{l}\text { Área a ser trabajada para o manejo correcto } \\
\text { de la micro cuenca }\end{array}$ & 78 & 8 & 1 & 10 \\
\hline 7 & Degradación de la micro cuenca & 76 & 8 & 1 & 10 \\
\hline \multicolumn{2}{|c|}{ Total de Factor Físico-Conservacionista } & $(1-7)$ & 38 & 7 & 70 \\
\hline
\end{tabular}

\begin{abstract}
Modelo matemático de la degradación ambiental.- El modelo matemático resultante expresado mediante la ecuación de la recta presentado según la ecuación 3.1 y la Figura 5, fue trabajado sobre la base de los valores significativos (encontrado, mínimo y máximo), presenta una inclinación angular de 1.587 y un coeficiente lineal de -11.111, dicha ecuación constituye el modelo matemático de la degradación ambiental físico-conservacionista de la sub-cuenca del rio Angasmarca. Según el valor significativo total encontrado para la sub-cuenca es de valor (38), Tabla7, podemos afirmar que la degradación ambiental es de $49.206 \%$, es decir, que el deterioro o degradación ambiental promedio encontrada en la subcuenca es de $49.206 \%$, es decir está por encima de los límites permisibles (encima del $10 \%$ ).
\end{abstract}

$$
Y=1.587 X-11.111(3.1)
$$

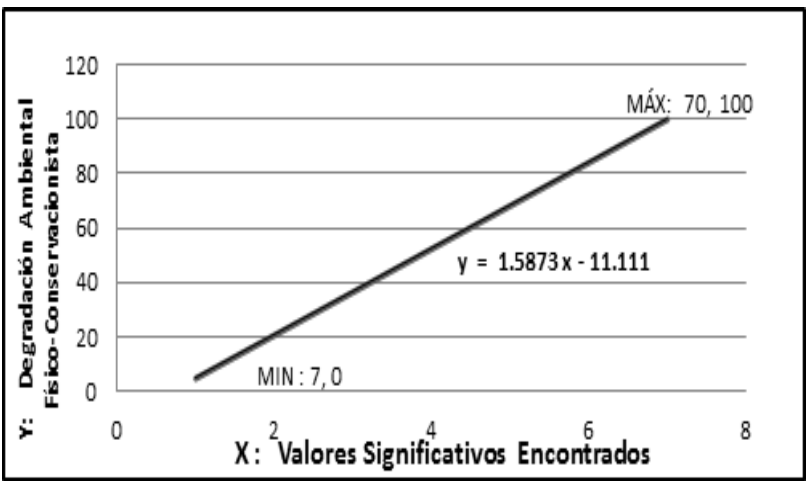

Figura 5. Recta de degradación ambiental FísicoConservacionista.

\section{Conclusiones}

Fue posible realizar un diagnóstico ambiental físicoconservacionista de la sub-cuenca del río Angasmarca a través de la evaluación de conflictos de uso del suelo y la determinación del grado de deterioro del medio ambiente, logrado mediante el uso de técnicas de Geoprocesamento, en la obtención de información georreferenciada, el modelado digital de terreno, caracterización morfométrica de micro cuencas hidrográficas, así mismo la obtención de la modelación matemática de la degradación ambiental. 
Las características o variables morfométricas de la sub-cuenca y micro cuencas del río Angasmarca correlacionadas con su geometría, relieve y red de drenaje indican que poseen formas un poco alargadas, lo cual indica que no están sujetas a inundaciones, pero que si favorecen a la escorrentía superficial.

La densidad de drenaje, indica baja capacidad de drenaje, la densidad hidrográfica es inferior de 1 curso/ $\mathrm{km}^{2}$ (se considerada baja) y las pendientes medias de las micro cuencas son altas e indican que la sub-cuenca está potencialmente sujeta a fenómenos de erosión, principalmente bajo condiciones de suelo expuesto o de escasa cubierta vegetal permanente.

En el análisis de los conflictos de uso del suelo, la evaluación ambiental físico-conservacionista de las micro cuencas 2, 7 y 9, están con la mayor degradación ambiental, con $89.16 \%$ y $76.48 \%, 100.00 \%$, respectivamente.

La degradación ambiental de la sub-cuenca Angasmarca es de 24,138.39 ha, equivalente al $68.37 \%$ del área total de la sub-cuenca. De dicho porcentaje de degradación el $84.39 \%$ se encuentra en micro-cuencas de clase A, el $13.87 \%$ en micro cuencas de clase B y solamente el $1.74 \%$ en micro cuencas de clase D.

El Modelo matemático de la degradación ambiental físico-conservacionista de la sub-cuenca hidrográfica del río Angasmarca, expresado mediante la ecuación de la recta, explica el grado de deterioro ambiental de la sub-cuenca y según el factor físico-conservacionista total encontrado la degradación ambiental promedio encontrado es de $49.206 \%$, lo cual se considera muy alto, es decir por encima de los límites permisibles (encima del $10 \%)$.

Es posible elaborar el modelamiento geográfico mediante técnicas de sensores remotos y de sistema de de información geográfica SIG, con integración del MDT, datos de uso potencial y de ocupación del suelo.

La evaluación ambiental físico-conservacionista y modelado matemático de la degradación ambiental de la cuenca hidrográfica, permite hacer un diagnóstico de la situación ambiental, identificando potencialidades de uso, ocupación, áreas en conflicto, en riego, vulnerabilidad o con limitaciones significativas, así mismo permite proponer acciones mitigadoras, como recomendaciones técnicas apropiadas para reducir impactos ambientales, estimar rendimientos futuros y tomar decisiones relativas a la conservación del medio y el desarrollo sustentable de la cuenca hidrográfica.

\section{Literatura citada}

Cabral, J. B. P. et al., 2005. "Estudo da erosividade e espacialização dos dados com técnicas de Geoprocesamiento na carta topográfica de Morrinhos Goiás/Brasil para o período de 1971 a 2000”, Geo Focus (Artículos), no 5, p. 1-18. ISSN: 1578-5157.
Guerra, A. y Cunha, S., 1996. Degradação ambiental, Geomorfologia e Meio ambiente, Rio de Janeiro: Bertrand Brasil, p. 337-379.

Iost, V. L., Teixeira, D., Costa, D. J. L. y Fuller, B. B. 2007. O Conceito de Bacia Hidrográfica e a Importância da Caracterização Morfométrica para o Entendimento da Dinâmica Ambiental Local. Ver. UNIARA, n.20, Pag. 147-157.

Horton, R., 1945. Erosional development of streams and their drainage basins: hidrophysical approach to quatitative morphology. New York: Geological Society of American Bulletin, New York, v.56, n. 3, p. 275-370.

Macedo, R. K., 1991. A importância da avaliação ambiental. In: TAUK, S. M. Análise ambiental: visão multidisciplinar. São Paulo /FAPESP/SRT/ FUNDUNESP, $169 \mathrm{p}$.

PERU, Ministerio de Agricultura-ANA, http://www. ana.gob.pe/marco-normativo.aspx, 2011. Acesso em 16/11/2011.

PERU, ONERN, 1972. Inventario evaluación y uso racional de recursos naturales de las cuencas hidrográficas de Santa Nepeña y Lacramarca, ONERN, Lima, Perú.

Rocha, J. S. y Kurtz, S. M. J. 2001. Manual de manejo integrado de bacias hidrográficas. $4^{\mathrm{a}}$ edição ampliada e melhorada. Santa Maria: Edições UFSM CCR/UFSM, 302 p.

Rocha, J. S. et al., 2008. Manual de EIA-RIMA com avaliações de impactos e passivos ambientais. $4^{\mathrm{a}}$. ed. rev. e ampl. - Santa Maria: Centro de Impressão Digital CID, xx, $479 \mathrm{p}$.

Tonello, K. C., 2005. Análise hidroambiental da bacia hidrográfica da Cachoeira das Pombas, Guanhães, MG. Viçosa: Universidade Federal de Viçosa, 85 p.

Vilela, S. M., Mattos, A. 1975. Hidrologia aplicada. São Paulo: McGraw Hill do Brasil, 245 p. 